\title{
Anatomical and technical factors associated with superior facet joint violation in lumbar fusion
}

\author{
Alisson R. Teles, MD, ${ }^{1-4}$ Michael Paci, MD, MScA, ${ }^{3}$ Gabriel Gutman, MD, ${ }^{1-4}$ \\ Fahad H. Abduljabbar, MBBS, ${ }^{1,2,5}$ Jean A. Ouellet, MD, ${ }^{1,2}$ Michael H. Weber, MD, PhD, ${ }^{1,2}$ and \\ Jeff D. Golan, MD',3,4 \\ ${ }^{1}$ McGill Scoliosis and Spine Group, McGill University Health Centre; Departments of 20 rthopedic Surgery and ${ }^{3}$ Neurosurgery, \\ McGill University; ${ }^{4}$ Department of Neurosciences, Jewish General Hospital, Montreal, Quebec, Canada; and ${ }^{5}$ Department of \\ Orthopedic Surgery, King Abdulaziz University, Jeddah, Saudi Arabia
}

\begin{abstract}
OBJECTIVE The aim of this study was to evaluate the anatomical and surgical risk factors for screw-related facet joint violation at the superior level in lumbar fusion.

METHODS The authors conducted a retrospective review of a consecutive series of posterior lumbar instrumented fusions performed by a single surgeon. Inclusion criteria were primary lumbar fusion of 1 or 2 levels for degenerative disorders. The following variables were analyzed as possible risk factors: surgical technique (percutaneous vs open screw placement), depth of surgical field, degree of anterior slippage of the superior level, pedicle and facet angle, and facet degeneration of the superior level. Postoperative CT scans were evaluated by 2 independent reviewers. Axial, sagittal, and coronal views were reviewed. Pedicle screws were graded as intra-articular if they clearly interposed between the superior and inferior facet joints of the superior level. Multivariate logistic regression analyses were conducted to assess the factors associated with this complication.
\end{abstract}

RESULTS One hundred thirty-one patients were included. Interobserver reliability for facet joint violation assessment was high ( $\kappa=0.789$ ). The incidence of superior facet joint violation was $12.59 \%$ per top-level screw (33 of 262 proximal screws). The rate of facet violation was $28.0 \%$ in the percutaneous technique group (14 of 50 patients) and $12.3 \%$ in the open surgery group (10 of 81 patients) (OR 2.26, 95\% Cl 1.09-4.21; $p=0.024)$. In multivariate logistic regression analysis, independent predictors of facet violation were percutaneous screw placement (adjusted OR 3.31, 95\% Cl 1.42-7.73; $p=0.006$ ), right-side pedicle screw (adjusted OR 3.14, 95\% Cl 1.29-7.63; $p=0.011$ ), and facet angle $>45^{\circ}$ (adjusted OR $10.95,95 \%$ Cl 4.64-25.84; $p<0.0001)$.

CONCLUSIONS The incidence of facet joint violation was higher in percutaneous minimally invasive than in open technique for posterior lumbar spine surgery. Also, coronal orientation of the facet joint is a significant risk factor independent of the surgical technique.

https://thejns.org/doi/abs/10.3171/2017.6.SPINE17130

KEY WORDS lumbar spine; minimally invasive surgery; facet joint; risk factors

$\mathrm{C}$ RANIAL facet joint violation has been an underappreciated consequence of pedicle screw placement in the lumbar spine. Injury of the facet joint at the level adjacent to a fusion is associated with alteration in the load-bearing capability of the segment, ${ }^{4,7}$ leading to accelerated degeneration of the joint ${ }^{17}$ and ultimately adjacent-segment disease (ASD). . $13^{1}$

The reported rates of facet joint violation at the top level (superior) vary from $0 \%$ to $100 \%$ in the literature. ${ }^{2,5}$, $6,8,9,11,14,15,18,22$ This wide variation is explained by the variability in surgical techniques, different image-guidance strategies, and different definitions of facet joint violation. Some authors have tried to identify risk factors for this complication. 2,6,9,11,14,18,22 However, the lack of controlling for confounders in the majority of these series has precluded this. For example, in a meta-analysis of the litera-

ABBREVIATIONS AP = anteroposterior; ASD = adjacent-segment disease; DFP = difference between facet and pedicle (angle); MIS = minimally invasive surgery; ROC = receiver operating characteristic.

SUBMITTED January 31, 2017. ACCEPTED June 6, 2017.

INCLUDE WHEN CITING Published online December 8, 2017; DOI: 10.3171/2017.6.SPINE17130. 
ture, Wang et al. ${ }^{20}$ found a rate of facet joint violation of $18.18 \%$ (154 of 847) in percutaneous screw placement and $18.72 \%$ (170 of 908) in open surgery. However, among series that involved minimally invasive percutaneous screw placement, 1 series used 3D-CT-based navigation and the other 3 used fluoroscopy. Also, the included studies used different grading criteria to evaluate joint violation.

The influence of the anatomy of the superior level in this complication is not well defined. Some authors have suggested that this complication could be more prevalent in lower levels of the lumbar spine (that is, L-4 and L-5). ${ }^{11,14,22}$ However, to the best of our knowledge, no study has identified the influence of anatomical variability of the facet joints in the occurrence of breaches. The objective of this study was to evaluate the anatomical and surgical risk factors for facet joint violation at the superior level of a lumbar fusion.

\section{Methods \\ Study Design and Patients}

After receiving institutional review board approval of the study protocol, we retrospectively reviewed a consecutive series of lumbar fusions performed between 2009 and 2016 at the Jewish General Hospital by a single surgeon (J.D.G.). Patients who underwent primary lumbar fusion of 1 or 2 levels for degenerative disorders during the study period were selected. Exclusion criteria were previous lumbar instrumentation, patients who did not undergo postoperative lumbar CT scanning, $>2$ levels of fusion, and if the reason for surgery was not degenerative disease.

\section{Surgical Technique}

The open technique was used mostly for patients with bilateral radicular symptoms. Fluoroscopy was used as image guidance for open and minimally invasive percutaneous screw placement.

In the open technique, a midline incision was used; subperiosteal dissection was performed down to the spinous processes, laminae, facet joint, and transverse processes of the chosen levels. The capsule of the superior facet of the upper-instrumented vertebra was left intact. Entry points for the pedicle screws were identified using surface landmarks and fluoroscopy. The pedicles were cannulated and adequate-sized screws were inserted at the chosen levels. After decompression, rods were inserted, standard decortication was performed to achieve fusion, and local autograft and allograft were incorporated.

In the percutaneous minimally invasive technique/ surgery (MIS), an anteroposterior (AP) view was used to place Jamshidi needles into the pedicles. The entry point was at the lateral-most aspect of each pedicle. The needles were advanced medially without breaching the medial pedicle cortex in AP view until they were noted to be within the vertebral body on the lateral view. K-wires were tapped in and left in position. Subsequently, a 3-cm longitudinal incision was made approximately $4 \mathrm{~cm}$ off midline on the side of the decompression, the fascia was opened, and the muscles were separated through tubular dilators to reach the selected facet and perform transforaminal lumbar interbody fusion. Percutaneous screws were placed using lateral fluoroscopy assistance. Rods and screw caps were inserted.

All patients' charts were reviewed to verify the technique used in each screw. In some cases, the pedicle was cannulated using direct visual guidance. Such cases were considered as an open technique, using a Wiltse approach, as opposed to a true percutaneous technique.

\section{Definition of Superior Facet Joint Violation}

As part of the surgeon's protocol, patients in whom lumbar spine instrumentation was placed underwent CT scanning before hospital discharge. The postoperative CT scans were evaluated by 2 independent reviewers (A.R.T. and M.P.), who were blinded to the surgical technique. Axial, sagittal, and coronal views were reviewed. A pedicle screw was graded as intra-articular if it was clearly traversing the joint and interposing between the superior and inferior facet joints of the superior level (Fig. 1). The top-level screws were classified as extra-articular if they did not traverse the joint (Fig. 2). Any discordance was resolved by consensus between reviewers after initial independent assessments.

\section{Predictors of Facet Joint Violation}

The following variables were collected during assessment of the immediate postoperative CT scan: depth of surgical field, defined as the distance between the L-4 lamina and skin, measured with sagittal cuts; degree of anterior slippage of the superior level, measured in millimeters; pedicle and superior facet angle, ${ }^{19,24}$ as well as the difference between facet and pedicle (DFP) angle, measured in the axial cuts for each side (Fig. 3). Facet degeneration of the superior level was measured using the immediate postoperative CT scan according to the Weishaupt classification: ${ }^{21}$ Grade 0 , normal; Grade 1, mild degeneration; Grade 2, moderate degeneration; and Grade 3 , severe degeneration.

\section{Statistical Analysis}

All statistical analyses were conducted with SPSS version 20.0. Categorical variables were described by their absolute (no.) and relative (\%) frequencies and continuous variables as the mean and standard deviation. Interobserver reliability was measured with Cohen's $\kappa$ coefficient for the definition of facet joint violation (intra-articular vs extra-articular). The Pearson correlation coefficient (r) was used for the classification of facet degeneration (Weishaupt classification). Interobserver reliability was high for facet joint violation assessment $(\kappa=0.789)$, and moderate for facet joint degeneration $(r=0.593)$.

For statistical purposes, each screw in the superior level was analyzed separately. Possible factors associated with superior facet joint violation were age, sex, year of surgery, side of screw, surgical technique (percutaneous vs open), level, number of levels (1 vs 2), depth of the surgical field (in $\mathrm{mm}$ ), degree of anterior slippage of the superior level (in $\mathrm{mm}$ ), pedicle angle, superior facet angle, DFP angle, and facet degeneration (analyzed as a continuous variable). Logistic regression with the backward method was used, and the final model maintained only the predictors 

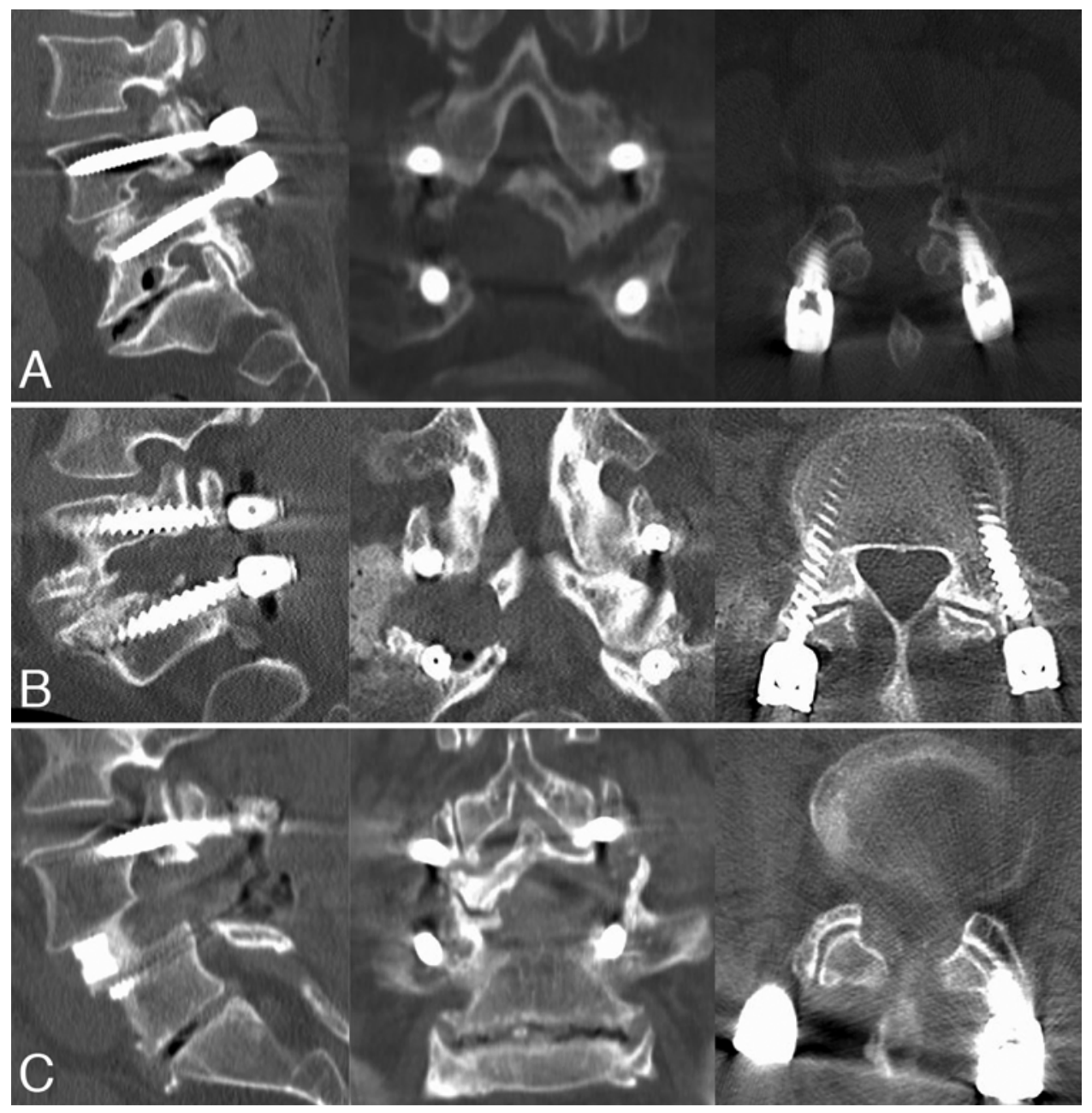

FIG. 1. Intra-articular screws were defined as screws that clearly traversed the facet joint and interposed between the superior and inferior facet joints of the superior level. Examples are shown of intra-articular screws at the superior level. A: Bilateral facet joint violation. B: Right facet violation. C: Left facet violation.

with a significance level $<0.05$ during the modeling steps. Variables included in the final model were age, number of levels, and superior level, because they were different according to the technique to control for these potential confounders. For anatomical features (pedicle angle, facet angle, and DFP angle), receiver operating characteristic (ROC) curve analyses were conducted to assess the variable most associated with facet violation. This variable was included in the final logistic regression model.

\section{Results}

During the study period, 149 patients underwent primary 1- or 2-level lumbar fusion for degenerative disorders, performed by the senior author (J.D.G.). After initial assessment, 18 patients (12\%) were excluded due to lack of postoperative CT scans. A total of 131 patients were included in this study. Among the 50 patients who underwent MIS, 3 had the proximal level screws inserted under direct visualization through the Wiltse approach instead of percutaneously (1 bilateral and 2 unilateral, 4 screws). Thus, a total of 262 proximal screws were analyzed: 96
MIS (percutaneous screw placement under fluoroscopy) and 166 under direct visualization (open technique). Comparison analyses demonstrated that patient groups were different in age, number of levels, and level of the superior instrumented vertebra (Table 1).
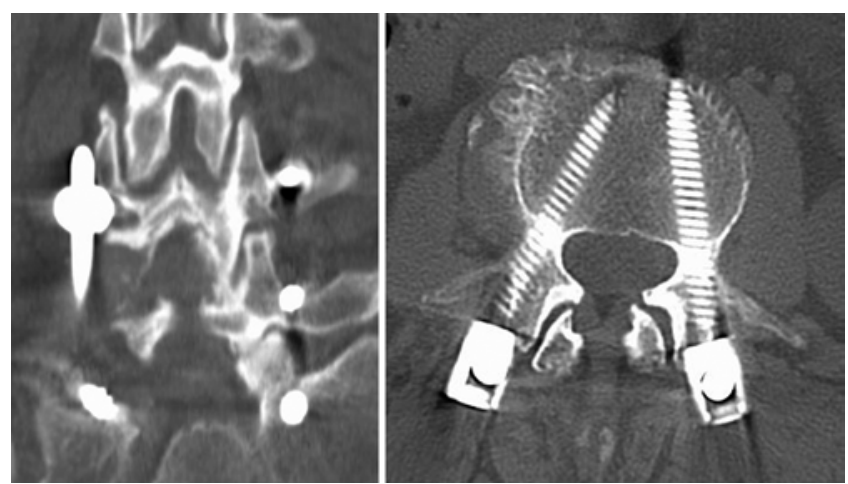

FIG. 2. Examples of screws at the top level that did not transverse the joints, which were considered extra-articular screws. 

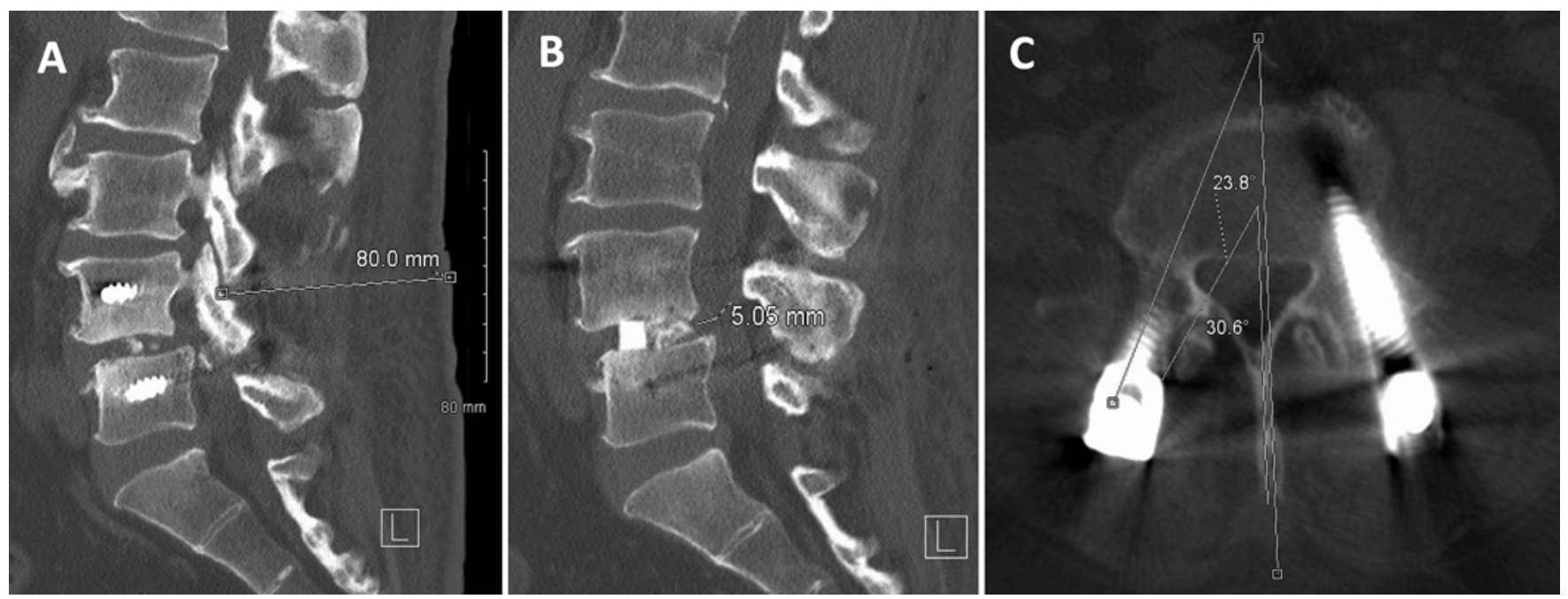

FIG. 3. Anatomical measurements in the CT scan. A: Depth of surgical field, defined as the distance between the L-4 lamina and skin. B: Degree of anterior slippage of the superior level, measured in millimeters. C: Pedicle and superior facet angle, as well as the DFP angle, measured in the axial cuts for each side.

\section{Superior Facet Joint Violation by the Pedicle Screw}

The incidence of superior facet joint violation was $12.59 \%$ per proximal-level screw (33 of 262 proximal screws). Of the 131 patients, 24 had superior facet joint violation (18.32\%); among them, 18 had unilateral facet joint violation and 6 had bilateral facet joint violation (5 MIS and 1 open surgery). The rate of facet violation was $28.0 \%$ in the MIS group (14 of 50 patients) and $12.3 \%$ in the open surgery group (10 of 81 patients) (OR 2.26, 95\% CI $1.09-4.21 ; \mathrm{p}=0.024)$. There were no screw-related complications requiring revision surgery during the early postoperative period.

\section{Factors Associated With Facet Joint Violation}

The results of univariate analyses of factors associated with facet joint violation are presented in Table 2. The variables age, MIS technique, right-side screw, top level at

TABLE 1. General features of the study sample

\begin{tabular}{lcccc}
\hline & & \multicolumn{2}{c}{ Screw Placement Technique } & $p$ \\
\cline { 3 - 4 } Variable & Total & Percutaneous & Open & Value \\
\hline No. of patients & 131 & 50 & 81 & \\
\hline Age in yrs & $60.66 \pm 13.17$ & $55.42 \pm 14.46$ & $63.16 \pm 12.82$ & 0.002 \\
\hline Female sex & $69(52.7)$ & $26(52.0)$ & $43(53.1)$ & 0.904 \\
\hline No. of levels & & & 0.026 \\
\hline 1 & $84(64.1)$ & $38(76.0)$ & $46(56.8)$ & \\
\hline 2 & $47(35.9)$ & $12(24.0)$ & $35(43.2)$ & \\
\hline Superior level & & & & 0.001 \\
\hline L-1 & $1(0.8)$ & 0 & $1(1.2)$ & \\
\hline L-2 & $3(2.3)$ & 0 & $3(3.7)$ & \\
\hline L-3 & $22(16.8)$ & $4(8.0)$ & $18(22.2)$ & \\
\hline L-4 & $85(64.9)$ & $31(62.0)$ & $54(66.7)$ & \\
\hline L-5 & $20(15.3)$ & $15(30.0)$ & $5(6.2)$ & \\
\hline
\end{tabular}

Values are presented as the mean \pm SD or as number $(\%)$.
L-4 or L-5, single-level fusion, higher degree of slippage, higher pedicle axial angle, higher superior facet angle, and higher DFP angle were associated with joint violation in the univariate analysis. The year of surgery was not associated with higher rates of facet violation (Fig. 4).

ROC curve analyses with the anatomical features showed that facet angle had a higher area under the curve (Fig. 5). Thus, this variable was considered for the final model in the logistic regression analysis. Figure 6 demonstrates the frequencies of intra-articular screws according to the superior level facet angle. It was observed that the greater the facet angle, the higher the likelihood of it being violated by the screw, independent of surgical technique.

After controlling for confounders, percutaneous screw placement (adjusted OR 3.31, 95\% CI 1.42-7.73; p = 0.006), right-side pedicle screw (adjusted OR 3.14, 95\% CI $1.29-7.63 ; \mathrm{p}=0.011$ ), and facet angle $>45^{\circ}$ (adjusted OR $10.95,95 \%$ CI 4.64-25.84; $\mathrm{p}<0.0001$ ) were independently associated with superior-level facet joint violation (Table 3).

\section{Discussion}

Adjacent-segment disease is an important cause of revision surgery after lumbar fusion, with an estimated rate of $1.8 \%$ per year. ${ }^{23}$ Several studies have identified risk factors for ASD including sagittal alignment, fusion length, age, fusion methods, presence of preexisting degeneration, previous surgery, and surgery-related violation of the adjacent segment. ${ }^{23}$ One of the factors potentially associated with ASD is violation of the superior facet joint by the pedicle screws.

In a biomechanical study, Cardoso et al. ${ }^{4}$ compared 4 models of L5-S1 fusion with different degrees of superior facet violation: 1) preserved L4-5 facets, 2) unilateral facet violation, 3) bilateral facet violation, and 4) bilateral facet violation and laminectomy of L-4. They showed a significant progressive increase in instability according to 
TABLE 2. Univariate analyses of factors associated with facet violation at the superior level

\begin{tabular}{|c|c|c|c|}
\hline Variable & $\begin{array}{c}\text { Intra-Articular } \\
\text { Screw }\end{array}$ & $\begin{array}{c}\text { Extra-Articular } \\
\text { Screw }\end{array}$ & $\begin{array}{c}p \\
\text { Value }\end{array}$ \\
\hline No. of screws & $33(12.6)$ & $229(87.4)$ & \\
\hline Age in yrs & $54.97 \pm 15.56$ & $61.06 \pm 13.01$ & 0.015 \\
\hline Sex & & & 0.374 \\
\hline Female & $15(10.9)$ & $123(89.1)$ & \\
\hline Male & $18(14.5)$ & $106(85.5)$ & \\
\hline Surgical technique & & & 0.001 \\
\hline MIS & $21(21.4)$ & $77(78.6)$ & \\
\hline Open surgery & $12(7.3)$ & $152(92.7)$ & \\
\hline Side & & & 0.041 \\
\hline $\mathrm{Rt}$ & $22(16.8)$ & $109(83.2)$ & \\
\hline Lt & $11(8.4)$ & $120(91.6)$ & \\
\hline No. of levels & & & 0.002 \\
\hline 1 & $29(17.3)$ & $139(82.7)$ & \\
\hline 2 & $4(4.3)$ & $90(95.7)$ & \\
\hline Superior level & & & 0.001 \\
\hline L-1 & 0 & $2(100)$ & \\
\hline L-2 & 0 & $6(100)$ & \\
\hline L-3 & 0 & $44(100)$ & \\
\hline L-4 & $21(12.4)$ & $149(87.6)$ & \\
\hline$L-5$ & $12(30.0)$ & $28(70.0)$ & \\
\hline Facet degeneration & $1.94 \pm 0.96$ & $1.93 \pm 0.83$ & 0.953 \\
\hline Depth, mm & $81.00 \pm 18.66$ & $76.87 \pm 15.57$ & 0.233 \\
\hline Slippage, mm & $4.64 \pm 3.36$ & $2.94 \pm 2.80$ & 0.009 \\
\hline Pedicle angle, ${ }^{\circ}$ & $19.61 \pm 4.37$ & $17.61 \pm 4.15$ & 0.011 \\
\hline Superior facet angle, $^{\circ}$ & $49.18 \pm 10.60$ & $34.93 \pm 11.17$ & $<0.0001$ \\
\hline DFP angle, ${ }^{\circ}$ & $29.57 \pm 9.65$ & $17.31 \pm 9.31$ & $<0.0001$ \\
\hline
\end{tabular}

All values are presented as the mean $\pm S D$ or as number (\%) unless otherwise noted.

degree of the superior-level violation. In addition, using a finite element analysis, Kim et al. ${ }^{7}$ showed that facet joint violation by pedicle screws leads to important increases in facet contact force and corresponding disc stress. Also, they demonstrated that the closer a pedicle screw is inserted to a facet joint surface, the greater the facet contact force caused by the pedicle screw.

Proietti et al. ${ }^{17}$ demonstrated an acceleration of facet degeneration in levels with screw encroachment 6-8 months after percutaneous lumbar screw placement. Wang et al. ${ }^{20}$ suggested that screw-related violation is a potential cause of postoperative pain due to impingement and instability. These reports highlight the importance of avoiding this complication during lumbar fusion. In this sense, to understand the potential risk factors would help surgeons to reduce the rates of superior facet violation.

The most common technique used for lumbar fusion is traditional open surgery through a midline incision. In this technique, careful preservation of the superior facet joint during the exposure and screw insertion is attempted. During the past decades, minimally invasive spine surgery has emerged due to its potential to reduce postoperative pain,

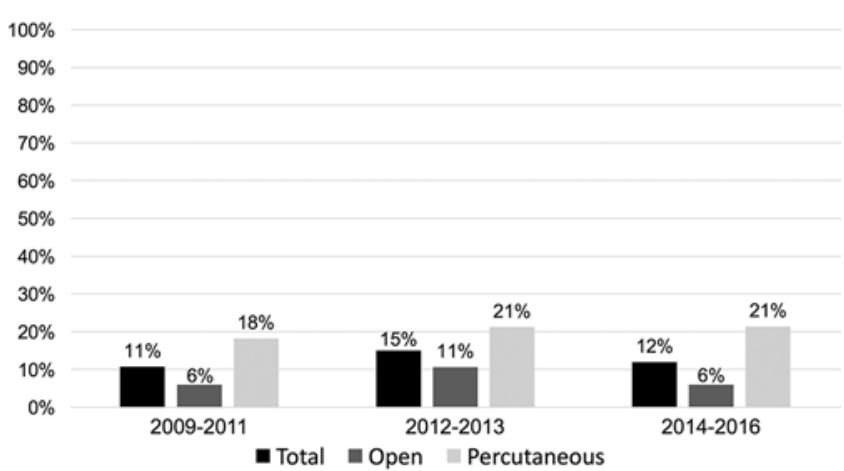

FIG. 4. Rates of superior facet violation according to the period of surgery. Note that there was no statistically significant difference in the rates of violation according to the period in the total sample (chi-square test $0.655, p=0.721$ ), open (chi-square test $1.072, p=0.585$ ), or percutaneous technique (chi-square test $0.224, p=0.894$ ).

blood loss, and duration of hospital stay, while achieving similar clinical outcomes compared with open surgery techniques. ${ }^{16}$ In addition, percutaneous screw placement may preserve the integrity of the facet joint by virtue of not dissecting it simply to identify the pedicle entry site.

One concern with this technique is the potential increase in the rate of screw-related facet joint violation of the top level due to difficult visualization of the joint. Park et al. ${ }^{14}$ reported the first study to evaluate the rate of superior facet joint violation by percutaneously placed pedicle screws in minimally invasive lumbar spine surgery. The authors showed incidence rates of $16.8 \%$ for facet joint violation in the superior level per screw and $28.3 \%$ per patient. Subsequent reports presented conflicting results on the influence of surgical technique (open vs percutaneous) and facet breach., 2,6,2,20,22 Our multivariate regression analysis demonstrated that screws inserted percutaneously were 3.31 times more likely to breach the facet independent of the anatomical features of the patient.

Our study showed that the orientation of the facets has a major impact on the rate of violation, independent of surgical technique (Table 3, Fig. 6). Previous studies have suggested the influence of the level on the rates of facet joint violation. Yson et al. ${ }^{22}$ identified a trend of increased rates of facet violation in lower levels. In the series of Park et al. ${ }^{14}$ the facet joint violation related to percutaneous pedicle screw placement occurred approximately 3.3 times more frequently at L5-S1 than at other levels. Also, Moshirfar et al ${ }^{11}$ reported a $48 \%$ rate of cranial facet joint violation at L-5 during open surgery. In our study, the variable that predicted this complication was the angle of the facet, rather than the level.

Other potential risk factors that have been suggested to influence the rates of facet joint violation were not confirmed by our analysis. For example, Babu et al. ${ }^{2}$ and Lau et al. ${ }^{9}$ suggested that obesity is associated with higher rates of facet violation. The rationale is that in obese patients, sometimes the correct entry point and trajectory are more challenging to obtain than in nonobese patients. We tested this hypothesis by evaluating the depth of the surgical field, defined as the distance between the L-4 lamina and the skin, and no association with facet violation was identified. 

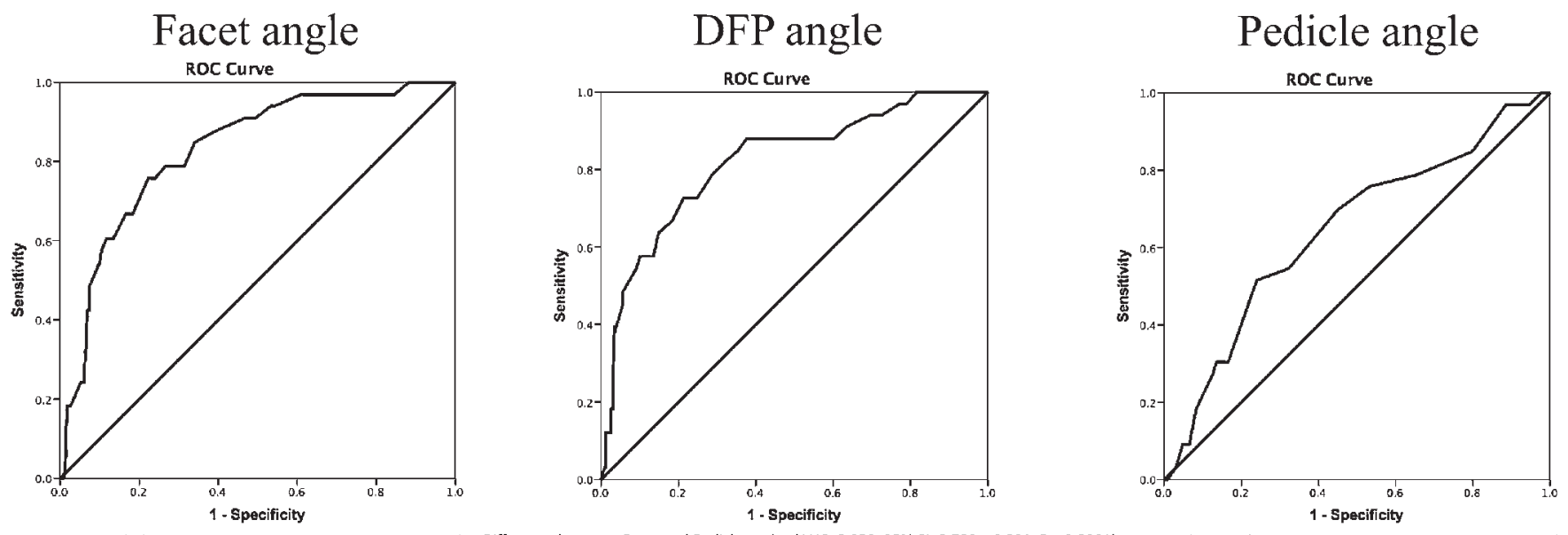

FIG. 5. ROC curve analyses on the vertebral anatomy predictors for superior facet joint violation.

In addition, we analyzed the influence of facet joint arthropathy on the occurrence of facet joint violation. In our series, we assessed facet degeneration using a validated classification, and no association was identified. The Weishaupt classification takes into consideration the joint degeneration, not the joint size. A larger facet may be harder to avoid with percutaneous techniques because it may be difficult to get lateral enough to avoid the facet and obtain appropriate medial angulation to cannulate the pedicle. Future studies could analyze the influence of this variable on this complication.

We identified that the screws on the right side of the patient were more likely to violate the joint independent of the surgical technique (open vs MIS) and anatomical features. This could be explained by the setup in the operating room in this series, in which the surgeon always stands on the left side of the patient and places screws from the contralateral side due to the bulkiness of the $\mathrm{C}$-arm machine on the right side. Another possible explanation is that some of the right-sided screws were inserted by trainees who were less experienced with the surgical procedure. However, in the study by Baird et al., ${ }^{3}$ no relationship between level of training and rate of superior facet violation was identified. We were not able to test this hypothesis due to the retrospective nature of the study. Park et al. ${ }^{14}$ suggested that surgical experience is associated with facet violation in MIS, with a trend toward reduction in the incidence as experience increases. In our study, there was no difference in the rate of facet violation over the years (Fig. 4).

Image guidance is essential for accurate percutaneous pedicle screw placement. In the study by Lau et al., ${ }^{9}$ the use of O-arm navigation did not seem to decrease facet violation rates compared with fluoroscopy (10.8\% with O-arm vs $4.8 \%$ with fluoroscopy). On the other hand, the study by Ohba et al. ${ }^{12}$ demonstrated lower rates of superior facet encroachment in cases that used intraoperative CT imaging navigation in MIS lumbar fusions (2.53\% with $\mathrm{O}$-arm vs $13.9 \%$ with fluoroscopy). An interesting study by Idler et al. ${ }^{5}$ reported the utility of a guided system based on accelerometer technology to facilitate obtainment of oblique

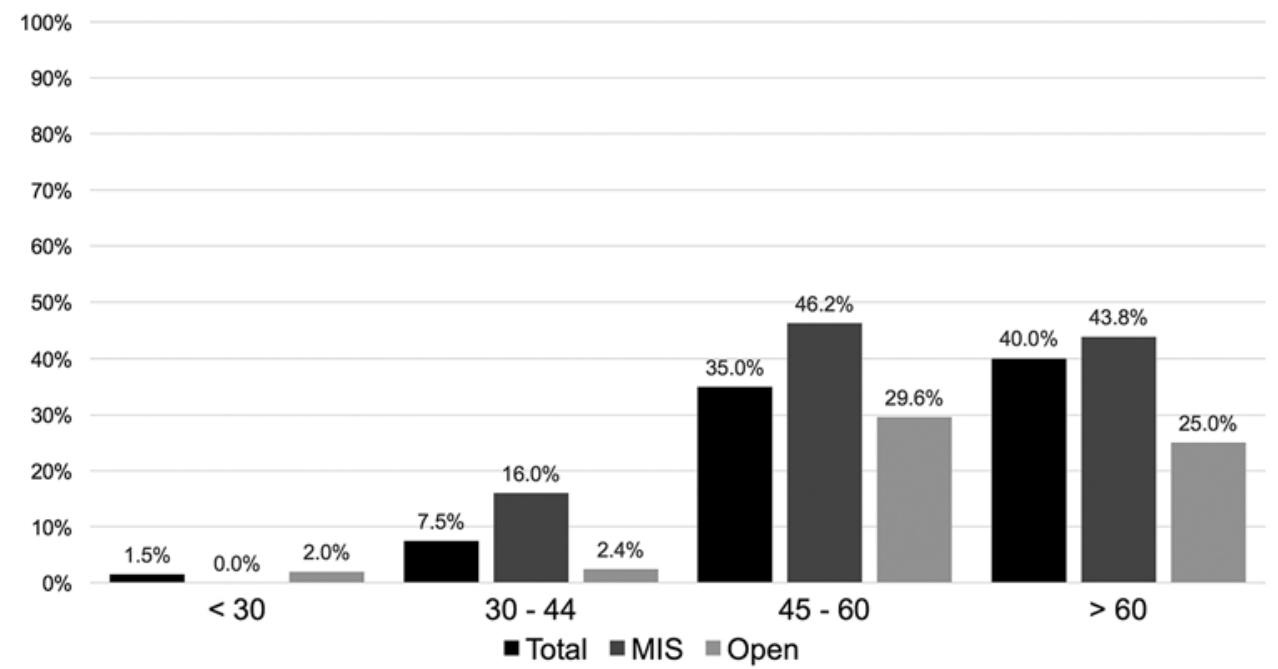

FIG. 6. Frequencies of intra-articular screws according to the superior-level facet angle. 
views with fluoroscopy for percutaneous screw placement. Using this technique, the authors reported no facet breach at the superior level in MIS lumbar fusions. Also, Matsukawa et al..$^{10}$ recently reviewed the incidence of superior facet violation in 202 patients who underwent lumbar fusion using the cortical bone trajectory technique with fluoroscopy and reported no intra-articular screw placement.

An audit of radiological and technical results is helpful to any surgeon and should be encouraged as a routine practice. Our study showed the results of a rigorous evaluation by 2 blinded reviewers demonstrating the incidence of screw-related joint violation and risk factors for this complication. These risk factors should raise surgeons' attention when performing lumbar spine instrumented fusions. These results influenced modification of the fluoroscopy technique used in our institution for a target view (oblique views), instead of purely AP and lateral views when performing percutaneous screw placement at superior levels.

Variables of patients included in the MIS and open surgery groups were different. Some of the differences are presented in Table 1 and were taken into account in the multiple logistic regression. However, one could argue that patients with unfavorable anatomy (that is, facet angles $>$ $45^{\circ}$ ) would have better radiological outcomes with open surgery. In our opinion, this conclusion is unfair because these patients could have had other drawbacks that were not considered in this study (e.g., hospital length of stay, opioid use, return to work, adjacent disease due to more involved tissue dissection to define entry points, and so on).

We recognize that our study has potential limitations. First, an MIS approach was more often used in single-level fusions, and consequently the top level was most likely L-4 or L-5. However, these differences were included in the final model of logistic regression to control for confounders. We determined that there was no relationship between the level and facet joint violation. Orientation of the facet joint was an independent predictor for facet joint violation. Second, not all patients had postoperative CT scans; however, this represented only $12 \%$ of patients who underwent 1- or 2-level lumbar fusion for degenerative disorders over a 7-year period.

Third, as a single-surgeon series, one could argue that the rates of violation may not apply for every surgeon. On the other hand, studies that report facet violation with multiple surgeons may lack standardization of technique. The benefit of a single-surgeon series is that it permits control for surgical technique, which was extensively described in the Methods. Furthermore, power analysis calculation indicates that this sample size (96 MIS screws vs 166 open surgery screws) has $80 \%$ of power ( $\beta$ error) to detect difference in the rates of facet violation, with both techniques at a level of significance of 5\% ( $\alpha$ error).

\section{Conclusions}

This study confirms the hypothesis that percutaneous screw placement using AP fluoroscopy to determine the pedicle entry site is associated with a higher likelihood of facet joint violation at the superior level in a lumbar fusion. Most importantly, we identified that the anatomy of the joint plays a major role in the occurrence of this complica-
TABLE 3. Logistic regression model of variables associated with Type 2 facet joint violation

\begin{tabular}{lcccc}
\hline \multicolumn{1}{c}{ Variable } & B value & Adjusted OR & $95 \% \mathrm{Cl}$ & $\mathrm{p}$ Value \\
\hline MIS & 1.19 & 3.31 & $1.42-7.73$ & 0.006 \\
\hline Facet angle $>45^{\circ}$ & 2.39 & 10.95 & $4.64-25.84$ & $<0.0001$ \\
\hline Rt side & 1.14 & 3.14 & $1.29-7.63$ & 0.011 \\
\hline
\end{tabular}

tion. Coronal orientation of the facets is a major risk factor for violation during pedicle screw placement. We strongly believe that an audit of radiological and technical results is helpful to any surgeon and should be encouraged as routine practice, even when clinical excellence (that is, no clinical complications such as screw revision) is presumed.

\section{References}

1. Aota Y, Kumano K, Hirabayashi S: Postfusion instability at the adjacent segments after rigid pedicle screw fixation for degenerative lumbar spinal disorders. J Spinal Disord 8:464-473, 1995

2. Babu R, Park JG, Mehta AI, Shan T, Grossi PM, Brown CR, et al: Comparison of superior-level facet joint violations during open and percutaneous pedicle screw placement. Neurosurgery 71:962-970, 2012

3. Baird EO, McAnany SJ, Overley S, Skovrlj B, Guzman JZ, Qureshi SA: Accuracy of percutaneous pedicle screw placement: does training level matter? Clin Spine Surg 30:E748E753, 2016

4. Cardoso MJ, Dmitriev AE, Helgeson M, Lehman RA, Kuklo TR, Rosner MK: Does superior-segment facet violation or laminectomy destabilize the adjacent level in lumbar transpedicular fixation? An in vitro human cadaveric assessment. Spine (Phila Pa 1976) 33:2868-2873, 2008

5. Idler C, Rolfe KW, Gorek JE: Accuracy of percutaneous lumbar pedicle screw placement using the oblique or "owl's-eye" view and novel guidance technology. J Neurosurg Spine 13:509-515, 2010

6. Jones-Quaidoo SM, Djurasovic M, Owens RK II, Carreon LY: Superior articulating facet violation: percutaneous versus open techniques. J Neurosurg Spine 18:593-597, 2013

7. Kim HJ, Chun HJ, Kang KT, Moon SH, Kim HS, Park JO, et al: The biomechanical effect of pedicle screws' insertion angle and position on the superior adjacent segment in 1 segment lumbar fusion. Spine (Phila Pa 1976) 37:1637-1644, 2012

8. Knox JB, Dai JM III, Orchowski JR: Superior segment facet joint violation and cortical violation after minimally invasive pedicle screw placement. Spine J 11:213-217, 2011

9. Lau D, Terman SW, Patel R, La Marca F, Park P: Incidence of and risk factors for superior facet violation in minimally invasive versus open pedicle screw placement during transforaminal lumbar interbody fusion: a comparative analysis. J Neurosurg Spine 18:356-361, 2013

10. Matsukawa K, Kato T, Yato Y, Sasao H, Imabayashi H, Hosogane N, et al: Incidence and risk factors of adjacent cranial facet joint violation following pedicle screw insertion using cortical bone trajectory technique. Spine (Phila Pa 1976) 41:E851-E856, 2016

11. Moshirfar A, Jenis LG, Spector LR, Burke PJ, Losina E, Katz $\mathrm{JN}$, et al: Computed tomography evaluation of superior-segment facet-joint violation after pedicle instrumentation of the lumbar spine with a midline surgical approach. Spine (Phila Pa 1976) 31:2624-2629, 2006

12. Ohba T, Ebata S, Fujita K, Sato H, Haro H: Percutaneous pedicle screw placements: accuracy and rates of cranial facet 
joint violation using conventional fluoroscopy compared with intraoperative three-dimensional computed tomography computer navigation. Eur Spine J 25:1775-1780, 2016

13. Park P, Foley KT: Minimally invasive transforaminal lumbar interbody fusion with reduction of spondylolisthesis: technique and outcomes after a minimum of 2 years' follow-up. Neurosurg Focus 25(2):E16, 2008

14. Park Y, Ha JW, Lee YT, Sung NY: Cranial facet joint violations by percutaneously placed pedicle screws adjacent to a minimally invasive lumbar spinal fusion. Spine J 11:295302, 2011

15. Patel RD, Graziano GP, Vanderhave KL, Patel AA, Gerling MC: Facet violation with the placement of percutaneous pedicle screws. Spine (Phila Pa 1976) 36:E1749-E1752, 2011

16. Phan K, Rao PJ, Kam AC, Mobbs RJ: Minimally invasive versus open transforaminal lumbar interbody fusion for treatment of degenerative lumbar disease: systematic review and meta-analysis. Eur Spine J 24:1017-1030, 2015

17. Proietti L, Scaramuzzo L, Schirò GR, Sessa S, Tamburrelli FC, Cerulli G: Degenerative facet joint changes in lumbar percutaneous pedicle screw fixation without fusion. Orthop Traumatol Surg Res 101:375-379, 2015

18. Shah RR, Mohammed S, Saifuddin A, Taylor BA: Radiologic evaluation of adjacent superior segment facet joint violation following transpedicular instrumentation of the lumbar spine. Spine (Phila Pa 1976) 28:272-275, 2003

19. Su BW, Kim PD, Cha TD, Lee J, April EW, Weidenbaum M, et al: An anatomical study of the mid-lateral pars relative to the pedicle footprint in the lower lumbar spine. Spine (Phila Pa 1976) 34:1355-1362, 2009

20. Wang L, Wang Y, Yu B, Li Z, Li Y: Comparison of cranial facet joint violation rate between percutaneous and open pedicle screw placement: a systematic review and meta-analysis. Medicine (Baltimore) 94:e504, 2015

21. Weishaupt D, Zanetti M, Boos N, Hodler J: MR imaging and CT in osteoarthritis of the lumbar facet joints. Skeletal Radiol 28:215-219, 1999
22. Yson SC, Sembrano JN, Sanders PC, Santos ER, Ledonio CG, Polly DW Jr: Comparison of cranial facet joint violation rates between open and percutaneous pedicle screw placement using intraoperative 3-D CT (O-arm) computer navigation. Spine (Phila Pa 1976) 38:E251-E258, 2013

23. Zhang C, Berven SH, Fortin M, Weber MH: Adjacent segment degeneration versus disease after lumbar spine fusion for degenerative pathology: a systematic review with metaanalysis of the literature. Clin Spine Surg 29:21-29, 2016

24. Zindrick MR, Wiltse LL, Doornik A, Widell EH, Knight GW, Patwardhan AG, et al: Analysis of the morphometric characteristics of the thoracic and lumbar pedicles. Spine (Phila Pa 1976) 12:160-166, 1987

\section{Disclosures}

Dr. Ouellet is a consultant for DePuy Synthes and has received clinical or research support for the study described (includes equipment or material) from AO Fellowship Funding.

\section{Author Contributions}

Conception and design: Teles, Golan. Acquisition of data: Teles, Paci, Golan. Analysis and interpretation of data: Teles, Golan. Drafting the article: Teles, Gutman. Critically revising the article: Gutman, Abduljabbar, Ouellet, Weber, Golan. Reviewed submitted version of manuscript: Teles, Abduljabbar, Ouellet, Weber, Golan. Approved the final version of the manuscript on behalf of all authors: Teles. Statistical analysis: Teles. Administrative/technical/material support: Ouellet, Weber, Golan. Study supervision: Ouellet, Weber, Golan.

\section{Correspondence}

Alisson R. Teles, 3801 Rue University, 109, Montreal, QC H3A 2B4, Canada. email: alisson.r.teles@gmail.com. 